\title{
Resultados de la interacción con la comunidad en la formación universitaria desde el enfoque de las capacidades. Un estudio de caso en la Universidad Nacional de Costa Rica
}

\section{Carmen Monge Hernández}

Universidad Nacional de Costa Rica.

Ingenio (CSIC - UPV), Universitat Politècnica

de València, España.

cmonge@una.cr

\author{
Priscilla Mena García \\ pris.mena@gmail.com
}

Rita Gamboa Conejo

rgamboa55@yahoo.com

Universidad Nacional de Costa Rica,

Costa Rica.
Investigación y extensión universitaria / Intervenciones
RECEPCIÓN: 05/12/17

ACEPTACIÓN FINAL: 21/06/18

\section{Resumen}

Este trabajo apunta al análisis de una experiencia de integralidad de las funciones académicas, sustentada en la extensión universitaria, que realiza la Escuela de Planificación y Promoción Social (EPPS) de la Universidad Nacional de Costa Rica (UNA). Nos proponemos aportar a la comprensión de algunos de los beneficios formativos conseguidos con el trabajo de Universidad y comunidad por los estudiantes de planificación económica y social a través de metodologías participativas, utilizando el enfoque de las capacidades para el desarrollo humano promovido por Sen y Nussbaum. En los resultados se destaca una gran riqueza en los procesos formativos, a partir de la relación dialógica, participativa, de interacción y construcción de saberes importante para el desarrollo humano de las personas. En la interrelación con los grupos más vulnerables y alejados de los beneficios de las políticas del Estado, mediante la puesta en práctica de los conocimientos teóricos y metodológicos adquiridos en la carrera y la realimentación con los otros saberes, se logran aprendizajes significativos que incrementan la sensibilidad, fortalecen los valores sociales y mejoran el análisis y la comprensión crítica de las necesidades sociales. Por lo tanto, los estudiantes tienen la oportunidad de tener una formación académica más integral y humanista.

Palabras clave: extensión universitaria, enfoque de las capacidades, desarrollo
Results of the interaction with the community in university education from the capabilities approach. A case study at the National University of Costa Rica

\section{Abstract}

This work aimed analyze an experience of how scholar actions work in an integral manner and based on the university extension done by the Escuela de Planificación y Promoción Social (EPPS) of National University, Costa Rica (UNA). Our purpose is to contribute on the understanding of some of the formation benefits derived from the university and community work done by economic and social planning students, using the capability approach for human development promoted by Sen and Nussbaum. The results highlight a great richness regarding to the formation processes based on a dialogical and participatory relation, as well as interaction and building up knowledge, all of which is important in human development. Students interact with the most vulnerable groups that are far from the State policy benefits through the methodologic and theoretical knowledge acquired on their formation and complemented with other knowledge. This promotes meaningful learning that increases sensibility, strengthens social values and improves analysis and critical comprehension of social needs. As a result, students have the opportunity of a scholar formation that is integral and humanistic.

Keywords: university extension, capability approach, human development
Resultados da interação com a comunidade na educação universitária a partir da abordagem de capacidades. Um estudo de caso na Universidade Nacional da Costa Rica

\section{Resumo}

Este trabalho se centraliza na análise de uma experiência integral das funções acadêmicas, fundamentada na extensão universitária, que realiza a Escola de Planejamento e Promoção Social (EPPS) da Universidade Nacional de Costa Rica (UNA). Nosso propósito é contribuir para a compreensão de alguns benefícios formativos conseguidos com o trabalho entre Universidade e Comunidade, realizado pelos estudantes de Planejamento Econômico e Social, utilizando metodologias participativas, focando-se nas capacidades para o desenvolvimento humano promovido por Sen e Nussbaum. Nos resultados se destaca uma grande riqueza nos processos formativos, a partir da relação dialógica, participativa, de interação e construção de conhecimentos importantes para o desenvolvimento humano das pessoas. $\mathrm{Na}$ inter-relação com os grupos mais vulneráveis e afastados dos benefícios das políticas do Estado, através da promoção dos conhecimentos teóricos e metodológicos adquiridos na formação profissional e na realimentação com os outros conhecimentos, atinge-se uma aprendizagem significativa que incrementa a sensibilidade, fortalece os valores sociais e melhora a análise e compreensão crítica das necessidades sociais. Portanto, os estudantes têm a oportunidade de ter uma formação acadêmica integral e humanista

Palavras-chave: extensão universitária abordagem de capacidades, desenvolvimento humano

Para citación de este artículo: Monge Hernández, C.; Mena García, P. y Gamboa Cornejo, R. (2018). Resultados de la interacción con la comunidad en la formación universitaria desde el enfoque de las capacidades. Un estudio de caso en la Universidad Nacional de Costa Rica. +E: Revista de Extensión Universitaria, 8(8), enero-junio, 110-125. doi: 10.14409/extensión.v8i8.Ene-Jun.7722. 


\section{Introducción}

Según Ruiz (1992), el ideario de la educación superior pública surge del legado de importantes líderes intelectuales de Latinoamérica y de la pugna de las clases sociales obreras. Con el antecedente de la Reforma de Córdoba (1918) inicia la nueva etapa de la vida universitaria al generar un planteamiento propio de universidad (Tünnermann Bernheim, 2000), democráticamente cogobernada, con altos estándares en el ámbito académico y de compromiso social y con vocación de democratización en el acceso y generación de conocimiento socialmente valioso (Arocena, 2010). Con la incorporación de la extensión como una nueva función en la universidad, junto a la docencia y la investigación, se promueve que los estudiantes tengan un acercamiento y comprensión crítica de la realidad sociocultural y política de las comunidades y que se propongan aportar a la transformación social. ${ }^{1}$ La extensión, desde una perspectiva no asistencialista ni transferencista, busca colocar la cultura como centro de la institución y satisfacer necesidades intrínsecas de la sociedad, entre ellas, preservar, desarrollar y promover la cultura (González et al., 2016). Se comprende que la educación y el conocimiento logran un "acto democratizador para la transformación y desarrollo social [en el que] se resignifica el propio concepto de misión, compromiso social y cultural de la universidad, donde la extensión adquiere un rol central en cada práctica transformadora" (Menéndez, 2017:29).

Por lo tanto, nuestro ideario es lograr que se favorezca, por medio de la extensión, un espacio de intercambio de saberes que beneficie tanto la vida de las personas como la propia Universidad. Es decir, procurar una transformación sociocultural, política y económica de la comunidad para tener mayores oportunidades de sostenibilidad y bienestar social, a la vez de mejorar la calidad de la formación universitaria.

Esta visión de integrar a la UNA con la comunidad surge desde su fundación, en 1973, cuando se concibió prioritaria la comunicación dialógica, cercana y permanente y de contribución sustantiva con la sociedad. En el caso de la UNA, encontramos que su modelo pedagógico considera la extensión como un componente fundamental de la academia. Así, la investigación, la extensión y la producción académica son las acciones que nutren la docencia (UNA, 2007). En esta misma orientación, en los Lineamientos para la Gestión de Programas, Proyectos y Actividades vigente se subraya que

"mediante su acción conjunta [de docencia, investigación y extensión] la Universidad Nacional devela los problemas fundamentales de la sociedad, propone alternativas y forma profesionales conscientes de las necesidades de la sociedad y del mundo en que vive". (UNA, 2009:5)

No obstante, no todos los programas de grado incorporan esta función; una de las escuelas líderes que lo hace es la de Planificación y Promoción Social (EPPS). El programa de grado se propone la integración del estudiante con la comunidad como parte de su proceso

1) En la extensión latinoamericana se destaca el trabajo realizado por importantes pioneros de la relación constructiva, crítica y dialógica con la comunidad, como son Paulo Freire y Orlando Fals Borda. El profesor Leopoldo Zea, de la UNAM México, subraya una tarea inquebrantable de la universidad pública latinoamericana, que no pierde vigencia, dirigida a mantener una universidad de carácter humanista y que busca constantemente brindar una formación que permita a las personas ser conscientes de su dignidad y partícipes activos en la problemática sociopolítica de su entorno (Ruiz Bravo, 1992). 
de aprendizaje. Por ello incorpora al currículo de grado cuatro cursos semestrales de naturaleza práctica, en el tercer año de carrera, denominados Práctica Organizativa I y II; y en el cuarto año, la Práctica de Formulación y Evaluación de Proyectos I y II. Para la UNA (1993), con estas acciones de participación del estudiante en la docencia, investigación y extensión, se promueve una formación integral, más humanista.

Los debates actuales a nivel global muestran las preocupaciones en torno a la presión que viven las universidades por parte del modelo normativo dominante. Esto está determinando que las universidades generen políticas y diseños pedagógicos que ponen el énfasis en la consecución objetivos basados en el interés del mercado de trabajo, la productividad, la mejora de ingresos, el liderazgo en los rankings internacionales, entre otros (Boni y Gasper, 2011; Walker, 2012). Nos referimos al modelo del capital humano que ejerce una gran influencia en la política educativa a nivel global y pone el sistema formativo al servicio de los intereses económicos (Walker, 2012). Por otro lado, estudios basados en el desarrollo humano centran la atención en la necesidad de crear capacidades y valores sociales que formen a los egresados tanto para que se desempeñen en el mercado laboral como para que promuevan transformaciones en favor del bienestar de las personas

Para lograr esa transformación real del estudiantado, durante el proceso formativo, los diseños pedagógicos y la formación de capacidades específicas resultan cruciales. Se busca, particularmente, que la educación superior se convierta en un espacio de enseñanza y aprendizajes capaz de generar una diferencia real en la vida del estudiantado y ampliar las oportunidades para los más vulnerables como, por ejemplo, para las mujeres y la clase obrera (Boni, 2013). Por lo tanto, un proceso formativo que integre el máximo de actores sociales en la construcción de oportunidades de desarrollo humano (Boni y Gasper, 2011), que potencie el aprendizaje experimentado (Belda et al., 2016) y la creación de pensamiento crítico y visión de largo plazo (Sousa Santos, 2005).

Entendemos que la extensión universitaria "promueve, a través de sus prácticas y acciones, una mejor calidad de vida, mayor inclusión y cohesión social y un desarrollo humano y sustentable" (Menéndez, 2017:30) y, por ende, apuesta a lograr un profesionalismo orientado al desarrollo humano. De allí que esta investigación explore, desde el enfoque de capacidades para el desarrollo humano, la experiencia del trabajo estudiantil en la comunidad durante la formación de grado de la carrera de Planificación y Promoción Social, con el fin de generar aportes que permitan profundizar la formación integral de los estudiantes. Se pretende avanzar en la comprensión de la contribución que generan las vinculaciones con las comunidades a la expansión de capacidades, en sus funcionamientos y la agencia de los estudiantes de la EPPS. Esto a fin de develar la riqueza académica, la relevancia de mantener estos cursos y de generar evidencias que aportan el trabajo estudiantil, sustentadas en la función de la extensión universitaria, que se desarrollan en Costa Rica y en otras latitudes. ${ }^{2}$

En este estudio de caso se aborda de manera cualitativa una experiencia de formación de las perspectivas de los estudiantes sobre los resultados logrados en dos cursos de comunicación con la comunidad.

2) Resultados preliminares de este análisis fueron discutidos en el III Congreso Internacional de Estudios del Desarrollo la Escuela de Ingeniería y Arquitectura de la Universidad de Zaragoza (España), realizado del 29 de junio al 1 de julio de 2016. 
Este artículo se propone presentar un primer apartado con un breve análisis del caso de la experiencia de la EPPS, seguido por los principales argumentos teóricos propuestos por el enfoque de capacidades como base de análisis. En el siguiente apartado, se hace referencia a los métodos utilizados $y$, en el tercero, se presentan los principales resultados obtenidos del análisis y la discusión, mostrando algunos de los más valorados por los estudiantes. Se finaliza con una breve reflexión académica de los resultados del estudio a modo de conclusiones.

\section{Experiencia de la carrera de Planificación Económica y Social en interacción con la comunidad}

El proceso formativo de esta carrera de grado, desde su origen en 1974, incluye cursos para que los estudiantes interaccionen con los sectores sociales. Los últimos cuatros planes de estudios contienen los cursos: Práctica Organizativa I y II, ambos de seis créditos, a partir del tercer año de la carrera. En una fase previa al desarrollo del curso, los profesores seleccionan espacios de demanda social en las comunidades rurales o urbanas y en instituciones públicas, para que el estudiantado ingrese y aborde realidades concretas. Un requisito básico es que exista aceptación por parte de la comunidad o de la institución de integrar un actor más, el sector académico (participación mínima de un profesor y varios estudiantes), para que le brinde el acompañamiento social y emprendan juntos el camino de construcción de oportunidades de desarrollo por medio de acciones de promoción y planificación participativa guiadas desde los cursos.

El objetivo del primer curso es desarrollar un diagnóstico participativo en conjunto con la comunidad, organización o institución, que permita la identificación de las principales problemáticas o necesidades sentidas por la población y la búsqueda consensuada de alternativas de solución realizables. Los estudiantes reciben instancias de formación previas al proceso de interacción con la comunidad que les posibilitan dialogar y participar activamente de sus actividades, promoviendo las condiciones para desarrollar el diagnóstico participativo que contemple aspectos ambientales, económicos, socioculturales y político-institucionales, lo que será la base para desarrollar el siguiente curso. Se pone especial énfasis en aspectos tales como el respeto a la cultura, la diversidad, las dinámicas comunales y sus propias visiones de desarrollo, entre otros. La EPPS dispone de una guía didáctica y su propia metodología para elaborar el diagnóstico participativo.

De acuerdo con EPPS (2016b), el curso de Prácticas Organizativas II fue diseñado con el propósito de desarrollar las acciones propuestas en el diagnóstico participativo, en procura mantener el mayor consenso posible por parte de los actores. Este curso da continuidad a la experiencia de construcción de conocimientos, desarrollo de habilidades, destrezas y procesos organizativos y de gestión social, todo ello orientado a promover acciones, proyectos o programas de desarrollo con visión integral. Con este curso se busca la construcción de una estrategia de desarrollo que permita contribuir al fortalecimiento institucional, comunitario y empoderamiento organizacional, así como al diseño, ejecución y evaluación de un subcomponente (proyecto/actividad) derivado de esa estrategia. El profesorado de estos cursos tiene la responsabilidad de promover la integración, profundización e interpretación de contenidos teóricos, metodológicos y técnicos, asumiendo así un rol de actor activo dentro de la ejecución de las acciones derivadas. 


\section{El perfil de profesionales deseado y las apuestas metodológicas para conseguirlo}

Hay una clara orientación de los cursos en función de los valores y logros profesionales y sociales de los participantes que se busca promover. Según el Informe evaluativo académico de la EPPS realizado en 1998, estos cursos constituyen un espacio orientado a formar un profesional capaz de desarrollar procesos socioorganizativos e institucionales que potencien la participación activa y comprometida de la sociedad civil, así como procesos de autogestión local y regional desde los propios actores del desarrollo. Ambos cursos propician acercamientos con la realidad nacional y promueven espacios constructivos con la sociedad. Para ello generan acciones o proyectos que favorezcan a la comunidad y a las organizaciones públicas y privadas. En el ámbito comunal, se potencia un trabajo con la sociedad civil, líderes locales y grupos organizados de base guiados a mejorar las condiciones socioeconómicas y ambientales en las que viven poblaciones vulnerables.

De acuerdo con EPPS, se pretende que

"el curso contribuya directamente en la vivencia de procesos sociales integrales, de tal manera que el estudiante además de la concepción epistemológica y teórica, entienda los procesos humanos y la transformación de la realidad a partir de la acción social con los otros. La participación es la clave fundamental de un proceso de democratización desde abajo". (2016a:2)

De manera que, aunque no aparece explícitamente en su programa de estudios, ambos cursos se suscriben al método de investigación-acción participativa, principalmente mediante las herramientas de la planificación participativa comunitaria. Si bien hay una orientación hacia la formación de los estudiantes, la acción va más allá, puesto que busca que las comunidades aporten al proceso formativo, convirtiéndose en formadoras desde sus conocimientos. Asimismo, a partir del diálogo y la participación, su objetivo no solo es la identificación de problemáticas, sino la priorización y búsqueda de medios para la consecución de logros importantes para la comunidad, desde una visión de desarrollo autónomo, centrado en el bienestar de las personas.

Esta experiencia favorece un modelo de trabajo universidad y sociedad generador de una cultura de diálogo y colaboración estrecha entre actores. Estos cursos "conciben el fortalecimiento organizativo como una condición para conseguir un avance en la capacidad de autoanálisis, autogestión de los grupos, así como la construcción conjunta del futuro deseado de todos los involucrados" (EPPS:1).

El modelo busca compartir visiones de desarrollo entre los participantes y aprovechar las metodologías participativas y herramientas de investigación para la construcción colectiva del conocimiento, de acciones desde los actores y la toma de decisiones consensuada, mediante una relación dialógica entre actores, donde la Universidad (el profesor y el estudiante) es un actor más. La expectativa académica es que este curso de aprendizaje y reflexión crítica "se convierta en una experiencia de vida" que marque positivamente a los participantes.

Los procesos de gestión social demandan mucho tiempo, esfuerzo, trabajo y compromiso. El desarrollo de los cursos exige una gran entrega por parte de los estudiantes, el profesorado y los miembros de la comunidad. Para el equipo de la Universidad, la presencia y seguimiento permanente en terreno o dentro de la organización requiere disponer y dedi- 
car al menos dos días por semana para el trabajo y la vinculación con la organización o la comunidad. Quienes realizan la práctica en sitios rurales alejados, ingresan a las comunidades un mínimo de dos veces por mes, según las condiciones de distancia, financiamiento, transporte y hospedaje, entre otras.

En ambos cursos se desarrollan sesiones de construcción grupal (taller) para el fortalecimiento de su práctica, del proceso metodológico y de técnicas a utilizar. Se brindan asesorías grupales, con una coordinación previa, que permiten la realimentación del profesorado respecto de contenidos y actividades. Se colabora en el abordaje del objeto de trabajo, en el análisis histórico, coyuntural y normativo, y en la elaboración de la estrategia de desarrollo y el impulso para el proyecto según se requiera. Cada profesor ofrece a su equipo de estudiantes el acompañamiento y la asesoría básica en las fases iniciales de la práctica para que ellos se afiancen y se garantice el compromiso y la legitimación de la UNA ante los principales actores sociales con los que interactúan así como de las iniciativas que se deriven del proceso.

Los cursos Práctica Organizativa I y II exigen que los estudiantes cumplan con todos los requisitos de cualquier curso universitario. El objetivo general de ambos cursos es "elaborar, en conjunto con una comunidad, organización o institución, un diagnóstico participativo que identifique las principales problemáticas y a partir de ese proceso, diseñar alternativa (s) de solución que se considere viable y factible" (EPPS, 2016b:3).

Para la elección de los espacios de trabajo compartido, históricamente se ha recurrido a aquellas comunidades $u$ organizaciones con las que se realizan proyectos de extensión universitaria de los diferentes programas de la EPPS. También se han logrado canalizar solicitudes de instituciones públicas o las demandas que transmiten comunidades socialmente vulnerables, sea mediante una comunicación directa (oral o escrita) o por medio de profesores, estudiantes, organizaciones comunales, sociedad civil, etcétera.

Una vez que se eligen las diferentes comunidades/organizaciones con las que se va a trabajar conjuntamente y comienza el ciclo lectivo, se realiza un diagnóstico de intereses y restricciones que poseen los estudiantes para desplazarse a las comunidades y se asigna el caso local u organización para que comience el trabajo en y con los actores vinculados. Para esto, los estudiantes, durante todo el año lectivo, trabajan cada quince días en y con la comunidad/organización donde están desarrollando sus prácticas y, por lo tanto, están en la obligación de permanecer al menos dos días en la comunidad (en esta experiencia, los días jueves y viernes). Para asegurar que el estudiante tenga la disponibilidad de responder favorablemente a este requerimiento y que logre cumplir con las exigencias de los cursos y de la comunidad, no se le permite matricular otras materias en esos días. Con esto se ha logrado que los estudiantes se dediquen por completo al desarrollo de las acciones de campo.

De manera adicional, los profesores responsables brindan semanalmente clases magistrales teóricas y asesorías prácticas en el aula y, como cualquier otro curso, se asignan lecturas complementarias durante el ciclo lectivo asociado al trabajo en comunidad. En estas clases los estudiantes reciben las orientaciones teóricas y metodológicas para elaborar el diagnóstico y el plan de acción comunitaria, análisis del comportamiento de los grupos, metodología de la investigación, contabilidad, análisis financiero, estadística, entre otros. Estos temas, complementados con otros cursos de su carrera, aportan la base para el trabajo con los grupos donde desarrollan sus prácticas. Es importante resaltar que la participación de los actores sociales es parte del propósito teórico, metodológico y epistemológico de es- 
tos cursos, en función de lo que la EPPS se ha planteado alcanzar en el propósito formativo. Lo que se busca es propiciar la generación de un diálogo de saberes entre la Universidad y la comunidad que logre aprendizajes significativos para ambas partes y una verdadera contribución a la creación de capacidades de los actores locales para encontrar alternativas sostenibles beneficiosas para su propio desarrollo.

El profesor tiene además la responsabilidad de efectuar visitas mensuales de supervisión del trabajo de campo para dar el debido seguimiento y realimentación a las diferentes acciones. En la evaluación de estos cursos se contemplan tanto los logros alcanzados en el diagnóstico y planteamiento de la estrategia de desarrollo participativa como los progresos logrados a nivel aptitudinal y actitudinal. Algunos de los criterios evaluativos incluyen: las contribuciones al equipo de trabajo y la integración con la comunidad u organización; capacidades de comunicación, convivencia y facilitación de procesos y construcción grupal; creatividad, motivación y compromiso. También se valoran la apropiación e identificación con las aspiraciones y cumplimiento de las expectativas comunales $u$ organizacionales, entre otros aspectos. La evaluación es un proceso donde también participan los actores locales con los que se interactúa como parte del reconocimiento de su contribución a la formación compartida con la Universidad.

\section{El enfoque de las capacidades y educación superior: conceptos clave que orientan esta investigación}

Esta investigación se desarrolla en la EPPS en el marco de la tesis doctoral que realiza la primera autora de este artículo en la Universidad Politécnica de Valencia en el tema de Desarrollo Local y Cooperación Internacional y en la línea de Cooperación internacional al desarrollo y territorio. El propósito es explorar los beneficios que se derivan de la integralidad de las funciones en términos de contribuciones a las capacidades del estudiantado así como disponer de información que sirva para realimentar las prácticas que realizamos como docentes. Las capacidades del estudiantado y sus condicionantes serán analizadas bajo la lupa del desarrollo humano. Por lo tanto, se utiliza el enfoque de las capacidades (capabilities approach) para el análisis, que es el enfoque que ha aportado al paradigma del desarrollo humano difundido por el Programa de las Naciones Unidas para el Desarrollo (PNUD) mediante los Informes de Desarrollo Humano. Este es un marco conceptual empleado en el ámbito normativo para la evaluación y el diseño del estado de bienestar individual y de las políticas de bienestar y de las instituciones, como son las universidades públicas y específicamente las acciones de integralidad de las funciones (Robeyns, 2017).

El enfoque de capacidades relaciona el desarrollo con las libertades de las personas (Sen, 1999). El desarrollo bajo este enfoque está vinculado con la libertad que poseen las personas para llevar la vida que valoran (ser y hacer), en donde la expansión de capacidades es el fin y el medio principal para el desarrollo. Este enfoque ofrece ventajas y oportunidades a la hora de cuestionarnos y enfocar las evaluaciones o juicios, considerando diferentes dimensiones (no basadas solo en lo cuantitativo o en el ingreso), mientras se observa y se reúnen datos, evaluando las vidas y las sociedades en que se desenvuelven (Robeyns, 2017). A partir de esto, resultan básicos tres conceptos de este enfoque: las capacidades, los funcionamientos y la agencia. 
Las capacidades (capabilities) representan el conjunto de libertades (oportunidades) que poseen los individuos para llevar la vida que valoran y que tienen razones para valorar. Los funcionamientos (functionings), por su parte, son las elecciones hechas por las personas o los logros alcanzados del conjunto de capacidades que disponen (Robeyns, 2003). De manera que las capacidades y los funcionamientos se pueden entender como lo que las personas pueden ser y hacer (sus capacidades, libertades u oportunidades reales) y lo que realmente están logrando en términos de seres y haceres/acciones (sus funcionamientos o el bienestar logrado) (Robeyns, 2017). La diferencia entre ambos conceptos está relacionada con que las capacidades constituyen el abanico de funcionamientos alcanzables de los que las personas pueden elegir, mientras que los funcionamientos son aquello que las personas realmente hicieron (por ejemplo, estudiar, descansar, alimentarse). Así, el hecho de que las personas tengan las capacidades (libertades) no significa que las utilicen o lleven a cabo las elecciones; cada persona puede hacer uso de sus capacidades de manera muy distinta. Aunque dos personas tengan capacidades similares, sus funcionamientos son diferentes, pues toman decisiones variables de acuerdo con su contexto.

Respecto de la agencia, se relaciona con la habilidad que posee la persona para alcanzar metas o valores sociales que considere importantes. Robeyns (2003) la vincula con aquella capacidad que permite que una persona sea capaz de provocar cambios en favor de otros. La autora apunta a que el bienestar personal y de contribución a otros genera la agencia global. Por lo tanto, el compromiso de esta acción de agencia no representa solo beneficio propio, para el individuo que impulsa la acción, sino para otros actores externos.

Nussbaum (2000) también realiza una contribución que aporta abundantemente a nuestro análisis. Este se refiere a las diez capacidades centrales para lograr una vida digna y que sirven de base para explorar las contribuciones desde la formación de la EPPS a los estudiantes, lo cual se presenta en el Cuadro 1.

Cuadro 1. Capacidades centrales para el funcionamiento humano.

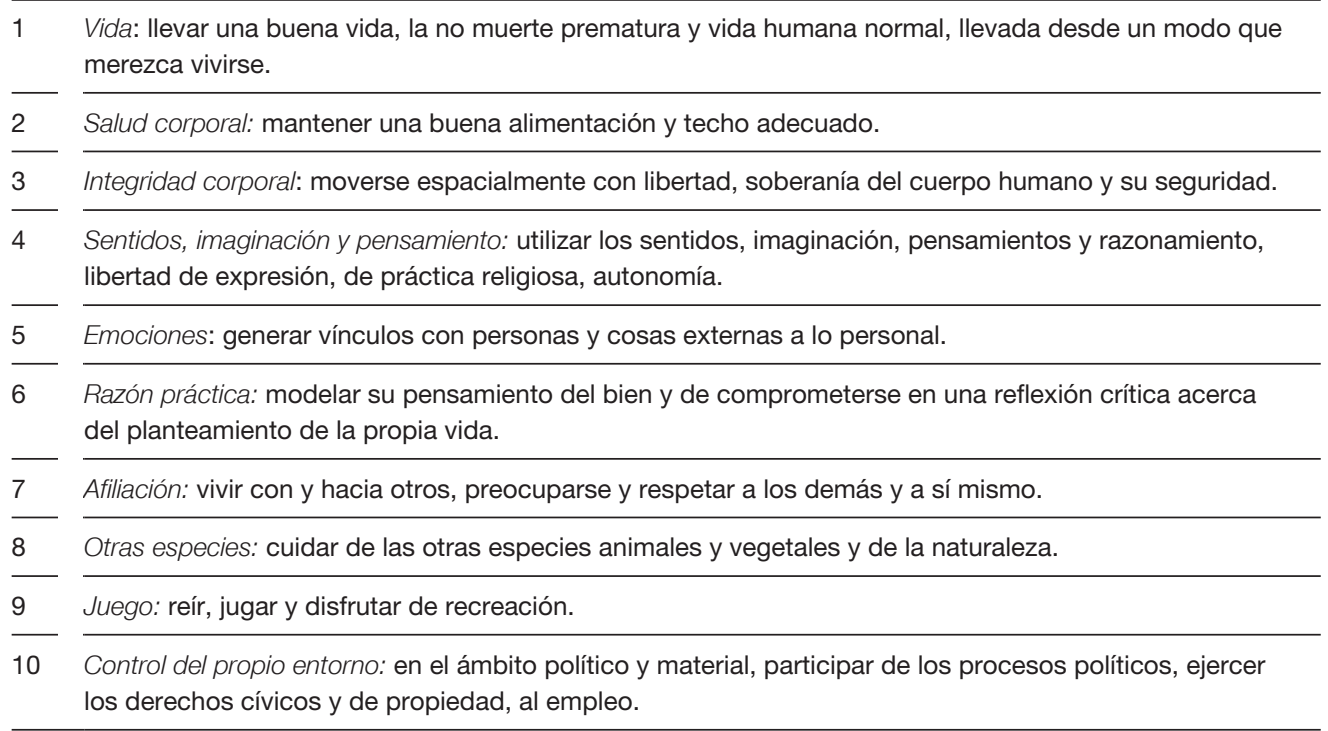

Fuente: elaborado a partir de Nussbaum (2000:120). 
Como el objetivo de este trabajo es comprender la contribución de los cursos mencionados a los estudiantes en términos de capacidades para el desarrollo humano, los aportes de este enfoque son importantes contribuciones para buscar y analizar evidencias sobre la potencial expansión de capacidades, funcionamientos y el incremento de agencia que viven los estudiantes de la EPPS a partir de los cursos de interacción con la sociedad. Por ello nos proponemos realizar una investigación exploratoria sustentada en las valoraciones que ofrezcan estudiantes activos en la carrera en contraste con otras fuentes de información.

\section{Metodología del trabajo}

Esta investigación busca describir, explicar y comprender los significados de la experiencia vivida acerca de las libertades que los estudiantes de estos dos cursos valoran como importante en su vida. Según Valles (1999), una investigación de este tipo se puede respaldar en la metodología cualitativa y se basa en el conocimiento que preserva la vida cultural, con la comunicación y la significancia que tienen los hechos para las personas. Para su abordaje, se parte de una experiencia que es sometida a una interpretación desde su contexto y de la perspectiva de los involucrados. Según este mismo autor, "no se buscan verdades últimas, sino relatos. El diseño está abierto a la invención; la obtención de datos al descubrimiento y el análisis a la interpretación" (Valles:56).

El punto de partida de esta investigación es la realidad y, según la epistemología del paradigma de investigación social constructivista interpretativo, ontológicamente se concibe que la realidad social es construida por los propios sujetos (Peris, 2014). Por lo tanto, el objeto de esta investigación lo constituyen los estudiantes de la EPPS de la Facultad de Ciencias Sociales, del Campus Omar Dengo de la provincia de Heredia de Costa Rica. En el Cuadro 2 se muestra el compendio de métodos aplicados, el cual incluye la realización de entrevistas semiestructuradas a 15 estudiantes y un grupo de discusión con otros 10 estudiantes, manteniendo consideraciones de confidencialidad, privacidad y consentimiento.

Cuadro 2. Compendio de métodos de recolección de datos.

\begin{tabular}{l} 
Método/Propósito \\
\hline Revisión documental: obtener la perspectiva institucional y documental de los cursos. \\
\hline Observación participante: comprender las experiencias, las relaciones y significancias de los procesos de los \\
cursos. \\
Entrevistas semiestructuradas y grupos de discusión: conocer los puntos de vista, opiniones, percepciones y \\
perspectivas de los estudiantes.
\end{tabular}

Fuente: elaboración propia.

Asimismo, para triangular información se efectuó una revisión documental y la observación participante mediante el acompañamiento a tres grupos de estudiantes en su trabajo en terreno. Se utilizan documentos no publicados como, por ejemplo, los dos programas de curso, los informes de cursos y tres diarios de campo de estudiantes del segundo semestre de 2015, pues dentro del curso I se utiliza la técnica de Diario de Campo Personal. 
El criterio de participación en la entrevista y los grupos de discusión es simplemente que hayan finalizado los cursos, los hayan aprobado o no. El grupo de discusión se realizó con 10 estudiantes que hicieron sus prácticas en diferentes partes del país y con la orientación de diferentes profesores.

\section{Análisis de resultados y discusión}

La información analizada, derivada de las técnicas aplicadas, nos ha permitido encontrar respuestas importantes en función de lograr la comprensión de beneficios de las prácticas de integralidad de las funciones dentro de la formación de grado, lo que presentamos a continuación.

Comenzamos concretando la perspectiva general que posee el estudiante sobre este segmento en particular de su formación, el período específico de navegación por la realidad social en terreno, con la interacción y el trabajo con la comunidad. Posteriormente, nos adentramos en la parte de resultados más importante, en términos de nuestros objetivos de investigación, cuando exploramos las capacidades y funcionamientos alcanzados por los estudiantes desde su propia perspectiva durante los cursos con el componente de integralidad de las funciones. Finalizamos con un breve análisis de las barreras o limitaciones del proceso que dan razón de la realidad que enfrentan los estudiantes durante esta experiencia y en donde podríamos centrar esfuerzos desde la UNA para contrarrestarlos y fortalecer las prácticas de extensión a futuro.

\section{¿Cuál es la valoración del estudiantado sobre su experiencia en comunidad?}

Los estudiantes coindicen en que ambos cursos permiten un espacio único durante la carrera para tener acercamiento a la realidad nacional y profesional, tanto en comunidades como al interior de algunas instituciones, situaciones que no se abordan desde las carreras universitarias. Estos cursos demandan mucho tiempo y exigen que el estudiante esté dedicado a la carrera dos semestres con la modalidad de tiempo completo. En el caso de que el curso fuese optativo, alrededor de la mitad de los estudiantes consultados revela que no lo cursarían porque es muy pesado en términos de dedicación de tiempo y esfuerzo. No obstante, en contraposición, encontramos otros estudiantes con la disposición voluntaria de elegir transitar ese camino, catalogándolo como un puente entre la teoría y la práctica y una fuente de creación de capacidades, por ejemplo, para formular correctamente ideas y estrategias participativas orientadas al bienestar social de los más necesitados.

\section{Nuevas capacidades y logros en los estudiantes con la interrelación con la comunidad}

En palabras de los estudiantes, "la práctica es una experiencia increíble" debido a que les permite conocer e interactuar con las distintas realidades sociales del país y comenzar la gestión integrada con las instituciones y organizaciones de apoyo social. A partir de la intervención en el territorio logran conocer diferentes procesos e interiorizar los problemas 
sociales desde la dinámica de las comunidades. Para quienes perciben de esta manera el proceso, esta experiencia constituye la primera oportunidad de ejercer su función profesional y llevar a la práctica los conocimientos adquiridos durante la carrera. Parte de los logros de los cursos de la práctica organizativa son aportar positivamente a la generación de posibles soluciones, resolver conflictos e involucrarse en procesos de cambio social y en la generación proyectos que favorecen el desarrollo del país, para que la gente acceda a mejores condiciones de vida. De manera que les ayuda a repensar la función social de su profesión.

Algunos de ellos expresan: "interactuar con personas diferentes me mejoró la visión de la realidad rural, este proceso me complementó lo aprendido en los cursos y libros" (Estudiante 7); "puse en práctica los conocimientos y conocí el ambiente social de una comunidad" (Estudiante 4); "mejoré mi percepción del desarrollo rural, tengo afinidad con el trabajo de campo con este tipo de población" (Estudiante 5); "aprendimos a relacionarnos con personas y nuevos conocimientos de la gente" (Estudiante 8).

Los estudiantes logran una mejor comprensión sobre los problemas sociales y de la realidad nacional porque se les permite adquirir información sobre las condiciones locales y la problemática que afrontan muchos lugares del país, las necesidades reales que tienen las personas, aquello en lo que es preciso trabajar para mejorar sus condiciones. Se reflexiona acerca de cómo este ejercicio les aclara qué tipo de persona y de profesional quieren ser realmente: "cambié al enfrentar la realidad social personalmente y no solo leerla de libros o artículos" (Estudiante 6); "no es lo mismo vivirlo que tenerlo en un libro" (Estudiante 8).

La aportación a la formación integral profesional es concebida desde la perspectiva de integración de los mismos en distintos ambientes de la sociedad, como se muestra en el Cuadro 3, lo que les permite aplicar el conocimiento adquirido durante la carrera y ampliar y fortalecer capacidades, habilidades y valores. En la convivencia con las comunidades y organizaciones, sus principales aprendizajes se desprenden al conocer directamente las condiciones de la zona específica, aprender de la vivencia de sus habitantes e identificar los deseos de superación que presenta cada comunidad o grupo a pesar de sus limitaciones.

Cuadro 3. Aportes a las capacidades personales y profesionales promovidas por los cursos Práctica Organizativa I y II de la EPPS según la perspectiva de los estudiantes.

\begin{tabular}{|c|c|c|}
\hline Ámbito personal & Ámbito profesional & Ámbito social \\
\hline $\begin{array}{l}\text { Cambio personal: al tener con- } \\
\text { ciencia sobre las realidades en la } \\
\text { que se desenvuelven gran canti- } \\
\text { dad de personas, los humaniza y } \\
\text { aspiran ser mejores. } \\
\text { Capacidades de resiliencia y re- } \\
\text { flexividad emocional: autoestima, } \\
\text { seguridad en sí mismos. } \\
\text { Fortalecimiento de valores: humil- } \\
\text { dad, respeto, responsabilidad, so- } \\
\text { lidaridad, libertad, constancia, } \\
\text { disciplina, etcétera. }\end{array}$ & $\begin{array}{l}\text { Nuevas habilidades: manejo de } \\
\text { grupos y comunicación. } \\
\text { Experiencia laboral valiosa para el } \\
\text { futuro. } \\
\text { Conocimientos para reali- } \\
\text { zar un trabajo académico de } \\
\text { manera participativa (métodos, } \\
\text { dinámicas, etcétera). } \\
\text { Comprender la demanda social y } \\
\text { ser más críticos de su realidad y } \\
\text { del tipo de desarrollo a promover. }\end{array}$ & $\begin{array}{l}\text { Conciencia social. } \\
\text { Compromiso con la acción social } \\
\text { para ayudar a los demás. } \\
\text { Sensibilidad con los problemas } \\
\text { sociales. } \\
\text { Criticidad a la hora de abordar las } \\
\text { problemáticas. } \\
\text { Activismo local y deseo de participa- } \\
\text { ción activa. }\end{array}$ \\
\hline
\end{tabular}

Fuente: elaboración propia con base en la metodología aplicada. 
Otros logros ponen en relieve el dominio de herramientas y nuevas capacidades básicas para su mejor desempeño profesional. Por ejemplo, en materia de investigación, al conseguir mejorar su desenvolvimiento para adquirir información y sus debidos procesos. En el domino en la aplicación de cuestionarios, entrevistas, herramientas de diagnóstico y talleres participativamente con las personas de las comunidades u organizaciones sociales. La adquisición de habilidades se respalda en expresiones como: "mejoré la comunicación, el trabajar en equipo y la creatividad (...) aprendí que la disciplina y la constancia son necesarias para legitimarnos en el proceso" (Estudiante 1); "aprendí del manejo de grupos, aprovechamiento de recursos y gestión de talentos" (Estudiante 2); "aprendí a saber escuchar, ser flexible, tener más facilidad para integrarnos y buen manejo de grupos" (Estudiante 10); "tengo más asertividad a la hora de comunicarme con los demás. Comprendí de la importancia de la cooperación y de las habilidades para negociar" (Estudiante 4).

La interacción con la comunidad también posibilitó el mejoramiento de su seguridad personal y autoestima, importantes para su profesión y muy necesarias para liderar procesos de participación social y el manejo de grupos. Por ejemplo, reducen el temor de hablar ante grupos de personas, amplían sus habilidades comunicativas y logran convencerse de su capacidad para ejecutar acciones con la comunidad y las organizaciones. El Estudiante 2 nos dice: "uno no está solo en el proceso de práctica [de trabajo con la comunidad], tiene al menos un compañero, ello da seguridad, pero también hay que confiar y creer en las capacidades personales, solo así se logra madurar como profesional". Por lo tanto, es una práctica que les ayuda a vencer la individualidad, por la que aprenden la importancia del trabajo en equipo y de saber delegar y confiar en los demás compañeros.

El conocimiento y soporte de la comunidad entregados al estudiante favorecen la generación de otras capacidades personales y profesionales. La confianza, la apertura, el afecto, las enseñanzas y la aceptación que reciben de parte de las comunidades pueden ser un importante insumo de mejoramiento de confianza y seguridad personal. El hecho de que la comunidad confíe en ellos, los considere un miembro más y participe activamente en todas las acciones ayuda a que los estudiantes se convenzan de sus habilidades y se sientan realmente capaces de liderar iniciativas de interés social. ${ }^{3}$

La interacción con contextos desconocidos hace que las personas aprendan a disfrutar de lo nuevo y a sobreponerse ante la adversidad. Algunas de las expresiones de los estudiantes también se podrían asociar a las capacidades de resiliencia y reflexividad emocional, como las siguientes: "aprendí que soy capaz de adaptarme a diferentes situaciones a las que uno no está acostumbrado" (Estudiante 1); "puedo superar retos como la escasez de recursos, el sufrimiento ajeno, considerando esto a través de ideas realistas pero humanas" (Estudiante 2); "logramos adaptarnos a escenarios difíciles y poder salir adelante (...) se aprende a complementarse con personas que actúan, se comportan y tienen actitudes muy diferentes a las nuestras" (Estudiante 3).

Ante la consulta de sus logros más valorados, funcionamientos en términos de Sen, llama la atención que se proyectan desde su función profesional y se observan algunos rasgos de

3) Esto pone de manifiesto la necesidad de realizar procesos de sensibilización con las comunidades para que comprendan las influencias de sus actitudes y entrega de saberes locales en los estudiantes. La educación no solo se promueve en las aulas universitarias, sino que se desarrolla en muchos espacios de comunicación con nuestros pueblos y nuestra cultura, por lo tanto, la comunidad es partícipe del proceso de enseñanza de los estudiantes. 
visión informada y de un cierto nivel de agencia. Expresan haber sido capaces de realizar los talleres con las personas locales y lograr que las personas se expresaran y ejercieran su participación. Tal es el caso del Estudiante 8, quien nos dijo: "la participación activa es clave para la defensa de los intereses comunes y colectivos, no individuales, y para su desarrollo hay que comprender las circunstancias, con el sentido analítico y la intuición". Esto se complementa con su satisfacción al haber conseguido la formulación de un trabajo (un proyecto) de manera conjunta con los diferentes actores locales para que se implemente una iniciativa en beneficio del bienestar colectivo y el desarrollo local. Para los estudiantes es muy importante lograr la participación social activa en la toma de decisiones y generar el apoyo y la cohesión social en torno a su desarrollo para dar respuesta a sus problemas más sentidos. Según el Estudiante 2, "la práctica permite cambiar la perspectiva y el enfoque, lo cual nos lleva a potenciar las metodologías".

La totalidad de los estudiantes considera que la experiencia de trabajar en las prácticas ha estimulado sus ideas, proyectos, actividades o acciones en defensa de sus propios derechos y de los de otras personas. Esto debido a que el proceso de práctica les ayudó a estar capacitados para orientar a grupos y formular ideas de proyecto que esperan aplicar también en su vida personal, familiar y comunal. Consideran que esta actuación en comunidad y con organizaciones puede ser considerada como una contribución para el mejoramiento del país. También comentan que han iniciado nuevos trabajos con varias asociaciones de desarrollo y el fortalecimiento de grupos dentro de las comunidades que les han solicitado información.

\section{Algunas barreras o limitaciones del proceso}

Las principales barreras que restringen sacar más provecho del curso están relacionadas con la necesidad de tener más recursos pedagógicos (por ejemplo, herramientas teóricas y preparación previo al trabajo de campo), pues se sienten un poco inseguros sobre la forma en que deben actuar y realizar el diálogo constructivo con las comunidades. Adicionalmente, piden la intermediación de la Universidad (del profesorado) para establecer una mejor relación con instituciones públicas como parte de su aspiración a lograr que las comunidades tengan acceso a mejores servicios públicos y de apoyo social. Reconocen que el profesorado debe darles más seguimiento durante las prácticas a nivel de coordinación de las actividades y la necesidad de disponer de alguna ayuda económica para desplazarse y permanecer más tiempo en las comunidades.

Dentro de sus preocupaciones también sobresalen la permanencia y la progresividad de las acciones. Según algunas opiniones, hacen falta información y compromiso con las personas vulnerables y los proyectos sociales, pues la mayoría de instituciones desconoce el trabajo que se está desempeñando y las necesidades sociales concretas (falta de caminos, de agua potable, de infraestructura comunal, de alternativas de entretenimiento, de capacitación, de mercadeo de productos agrícolas, etc.). ${ }^{4}$ Por último, en el ámbito económico es necesario resolver el financiamiento de materiales, transporte y alimentación durante los días que permanecen

4) Lo que los estudiantes perciben como una barrera para lograr un mayor impacto de su trabajo en la comunidad puede tomarse como evidencia de las necesidades que tienen actualmente las comunidades costarricenses y las organizaciones sociales y de las limitaciones que esto representa para su desarrollo. Lo que además respalda la vigencia y relevancia de la extensión universitaria en las comunidades. 
en la comunidad. Tienen algunas dificultades de traslado, ya que la mayoría de los trabajos se desarrollan en comunidades muy alejadas del Gran Área Metropolitana (GAM).

\section{Reflexiones finales}

En los dos cursos de grado de la carrera de la EPPS analizados se está logrando integrar los tres pilares de la academia (docencia, investigación y extensión) y, por lo tanto, sostener trabajos estudiantiles en y con la comunidad que dan razón de los beneficios de la integralidad de las funciones. Si bien es cierto que se trata solo de dos cursos de una carrera específica, este espacio representa un medio y una oportunidad para demostrar la pertinencia de la integración de la investigación y de la extensión en la docencia, lo que favorece la formación integral de los estudiantes y además es una contribución a las diferentes poblaciones costarricenses y a la realimentación de la academia, tema que esperamos abordar en un futuro estudio. Esta investigación ha permitido identificar una serie de argumentos sobre la contribución a la formación universitaria desprendida de las interrelaciones de la Universidad con la comunidad en función de la promoción de las capacidades centrales para una vida digna de Nussbaum (2000). Encontramos una especial orientación a la creación capacidades vinculadas con los sentidos, imaginación y pensamiento, la razón práctica, la afiliación y el control del propio entorno. El trabajo en y con la comunidad desarrollado por los estudiantes también aporta a la promoción de su capacidad de agencia, ya que ellos muestran progreso en su interés, convicción y habilidades para impulsar acciones en favor del bienestar de las poblaciones que necesitan de su apoyo.

La integralidad de las funciones favorece que los estudiantes avancen en la comprensión sobre la importancia de la participación y la autonomía local como motor de desarrollo y de cambios sociales sustantivos para el bienestar de la gente. Ellos comprenden los beneficios del trabajo social, especialmente cuando se trabaja de "abajo hacia arriba" (desde las personas, desde la comunidad, desde el tejido social hacia los gobiernos locales y las organizaciones públicas y privadas), integrando las voces de las personas y las organizaciones locales y de apoyo social. Asimismo, al pasar por esta experiencia, los estudiantes muestran progreso en la sensibilización ante las realidades ajenas, la empatía, los valores sociales y la contribución de la comunicación con los más vulnerables y, por ello, un mayor carácter humanista de la profesión.

Esta experiencia no solo contribuye a su formación profesional en términos utilitaristas, sino que aporta a la creación de capacidades humanas que impactan positivamente sus vidas, que se evidencian en aspectos como integridad, autoestima, seguridad en sí mismos y reflexividad emocional. Desde la perspectiva del estudiantado, el valor de esta experiencia se advierte en aspectos que les servirán para toda su vida, en lo profesional y en lo personal, en el incremento de las capacidades para comprender las necesidades reales de las poblaciones, en el trabajo colectivo, para reflexionar y construir alternativas de desarrollo desde, con y para la gente, mediante metodologías altamente participativas. Esto habla de cómo los estudiantes logran capacidades y funcionamientos para escuchar y reflexionar sobre las problemáticas y para conducir acciones basados en el sentir de la gente, lo que favorece la mejora de condiciones locales y el cambio hacia el desarrollo humano de nuestra sociedad. 
El hecho de transitar por la realidad social ha permitido a los estudiantes consultados potenciar los conocimientos teóricos y metodológicos adquiridos en el aula al llevarlos a la acción. Igualmente, con este ejercicio de interacción Universidad y comunidad se han logrado aprendizajes significativos que son sumamente valiosos en su proceso de formación. Este diálogo constituye una oportunidad para fortalecer y crear capacidades, validar y adaptar los conocimientos y complementarlos con los saberes de la gente.

La extensión, lejos de ser una receta, se convierte en un proceso de diálogo y construcción permanente que se lleva a cabo según las necesidades y prioridades de la población. Lo que además supone desafíos académicos de recuperar y sistematizar esas adaptaciones y nuevos conocimientos que surgen desde la interacción con la comunidad.

\section{Agradecimientos}

Este trabajo se dedica a las diferentes comunidades de nuestro pequeño país, Costa Rica, que desde inicios de la carrera de Planificación Económica y Social, hace más de 40 años, nos han abierto sus puertas para dialogar y construir procesos participativos en las cuatro dimensiones del desarrollo: sociocultural, económica, político-institucional y ambiental. A los profesores visionarios de la EPPS que han luchado por salir del espacio físico de la Universidad en búsqueda de la realidad, prácticas que aún permanecen en las aulas dando soporte al trabajo con las comunidades durante más de 30 años. Nuestro reconocimiento, por supuesto, a los estudiantes que día a día, con su vitalidad, responsabilidad y creatividad, renuevan nuestro compromiso social en este camino de construcción por un desarrollo local participativo e integral con los más necesitados y que, desde su imaginario, aportaron información para desarrollar esta investigación.

\section{Referencias bibliográficas}

Belda-Miquel, S.; Blanes, J.P.; Fernández, I.C. (2016). Participation for transformative learning in development management: The case of a Master in international development projects in the Universitat Politècnica de València (Spain). Procedia-Social and Behavioral Sciences, 228, 237-242.

Boni, A. (2013). \&- first century. In Boni, A. \& Walker, M. (Eds.). Universities and Human Development. A New Imaginary for the University of the XXI Century. London: Routledge.

Boni, A. y Gasper, D. (2011). La Universidad como debiera ser. Propuestas desde el desarrollo humano para repensar la calidad de la Universidad. Sistema. Revista de Ciencias Sociales, (220), 99-115

EPPS (2016a). Programa del curso Práctica Organizativa I. Facultad de Ciencias Sociales. Heredia, Costa Rica. (2016b). Programa del curso Práctica Organizativa II. Facultad de Ciencias Sociales. Heredia, Costa Rica. González, M.; Portero, A.E.; Sáez, H.T.; Jardo, R.M.; Fariñas, M.C. (2016). La práctica integral de arquitectos. Experiencia en la CUJAE. Estoa. Revista de la Facultad de arquitectura y Urbanismo de la Universidad de Cuenca, 5(8), 93-100.

Menéndez, G. (2017). Resignificación de la extensión a 100 años de la Reforma Universitaria de 1918. Revista + E, 7(7), 24-37. Santa Fe, Argentina: Ediciones UNL.

Nussbaum, M. (2000). Women and human development: The capabilities approach. Cambridge: Cambridge University Press. 
Peris, J. (2014). Aproximación a las metodologías cualitativas. Máster en Cooperación al Desarrollo. Universidad Politécnica de Valencia, España.

Robeyns, I. (2003). The capability approach: an interdisciplinary introduction, teaching material for the raining course preceding the 3rd International Conference on the capability approach. Pavia, September.

(2017). Wellbeing, freedom and social justice: The capability approach re-examined. Open Book Publishers.

Ruiz Bravo, R.M. (1992). Hacia una difusión cultural universitaria. Heredia, Costa Rica.

Sousa Santos, B. de (2005). La universidad en el siglo XXI. Para una reforma democrática y emancipadora de la universidad. Umbrales, (15), 13-70.

Sen, A. (1999). Development as Freedom. New York: Knopf.

Tünnermann Bernheim, C. (2000). Universidad y sociedad. Balance Histórico y Perspectivas desde Latinoamérica. Comisión de Estudios de Postgrado. Facultad de Humanidades y Educación. Universidad Central de Venezuela.

UNA (1993). Estatuto Orgánico de la Universidad Nacional. Aprobado por la Asamblea Universitaria mediante Referéndum realizado el 10 de marzo de 1993, transcrito por el Tribunal Electoral en comunicado oficial 03-93.

(2007). Gaceta Universitaria № 2: Modelo Pedagógico de la Universidad Nacional. Heredia, Costa Rica.

(2009). Lineamientos para la Gestión de Programas, Proyectos y Actividades. Gaceta Universitaria № 17-2009. Consejo Universitario. Costa Rica.

Valles, M.S. (1999). Técnicas cualitativas de investigación social. España: Síntesis.

Walker, M. (2012). A capital or capabilities education narrative in a world of staggering inequalities? International Journal of Educational Development, 32(3), 384-393.

Walker, M.; McLean, M. (2013). Professional education, capabilities and the public good: The role of universities in promoting human development. London: Routledge. 